\title{
The Role of Genetic Counselors for Patients with Familial Hypercholesterolemia
}

\author{
Amy C. Sturm
}

Published online: 19 April 2014

(C) Springer Science + Business Media New York 2014

\begin{abstract}
Familial hypercholesterolemia (FH) is the most common inherited cause of premature coronary heart disease and leads to significant morbidity and mortality. Genetic counselors are specialized health care professionals with advanced degrees and training in both medical genetics and psychosocial counseling. FH patients and their families require focused education regarding the heritable nature of their disease, the risk to family members, the necessity of cascade screening, and the availability of genetic testing. Patients and families may also benefit from additional services genetic counselors provide, such as psychosocial support and other resources. All of these services are well-established components of the genetic counseling process. Therefore, genetic counselors can serve as a valuable resource to FH patients, their families, and the multidisciplinary team of clinicians providing care for these patients.
\end{abstract}

Keywords Familial hypercholesterolemia - Genetic counselor · Genetic counseling · Cascade screening . Cascade testing $\cdot$ Genetic testing

\footnotetext{
A. C. Sturm $(\bowtie)$

Division of Human Genetics, Department of Internal Medicine, Ohio State University Wexner Medical Center, 2001 Polaris Parkway, Columbus, OH 43240, USA

e-mail: amy.sturm@osumc.edu

\section{A. C. Sturm}

Dorothy M. Davis Heart and Lung Research Institute, Ohio State University Wexner Medical Center, 473 W. 12th Ave.,

Columbus, OH 43210, USA
}

\section{Introduction}

Familial hypercholesterolemia $(\mathrm{FH})$ is a common inherited disorder associated with a very high risk for premature coronary artery disease (CAD). FH is treatable, but effective therapy requires early clinical diagnosis, and $\mathrm{FH}$ is currently vastly underdiagnosed and therefore undertreated $[1 \bullet \cdot$. In order to identify new cases of FH, it is imperative to initiate cascade testing after an index patient with $\mathrm{FH}$ has been diagnosed. This article specifically focuses on the potential role of the genetic counselor for patients and families with $\mathrm{FH}$ and provides information regarding the way in which these specialized healthcare providers may be able to serve as a resource to clinicians caring for and managing patients with $\mathrm{FH}$.

\section{Familial Hypercholesterolemia}

FH is the most common genetic cause of premature coronary artery disease (CAD) and is due to lifelong elevated plasma low-density lipoprotein cholesterol (LDL-C) levels. FH is autosomal dominant with a gene dosage effect with homozygotes (HoFH) or compound heterozygotes having a much higher LDL-C and much earlier CAD onset than heterozygotes (HeFH). The prevalence of $\mathrm{HeFH}$ in most Caucasian populations is estimated as 1:250-1:500; in specific founder populations, such as French-Canadians in Quebec, the prevalence of HeFH is higher [2]. HoFH is much rarer, with a prevalence of $1: 160,000$ to $1: 1,000,000[1 \bullet \cdot$. In untreated patients with $\mathrm{HeFH}$, there is a 20 -fold increased risk of premature CAD with the cumulative risk of fatal and nonfatal coronary events being $50 \%$ in men by age 50 and $30 \%$ in women by age 60 years [3]. In some patients, cholesterol deposits 
can be found in the tendons (xanthomas) and/or around the eyes (xanthelasmas).

Three sets of diagnostic criteria for HeFH exist: the US Make Early Diagnosis Prevent Early Death (MEDPED) [4], the Dutch Lipid Clinic Network [5], and the Simon Broome Register criteria [6]. The MEDPED criteria mainly rely on age-specific LDL-C levels and family history of FH [4]. The Dutch Lipid Clinic Network and Simon Broome Register criteria both involve a combination of LDL-C levels, physical features, family history, and the presence of a gene mutation $[5,6]$. These diagnostic criteria should only be used for FH index case identification; lower LDL$\mathrm{C}$ cut points can be applied to identify affected relatives.

There have been promising developments in the area of FH treatment. While statins should be the first-line therapy for patients with $\mathrm{FH}$, a significant number of patients are unable to reach their recommended LDL-C target without additional and/or alternative treatments, while others are unable to tolerate statins [2, 7]. Excitingly, the US Food and Drug Administration recently approved new therapeutic agents for $\mathrm{HoFH}$, including mipomersen and lomitapide [8]. Mipomersen has also shown great promise for $\mathrm{HeFH}$ patients with coronary artery disease and severe hypercholesterolemia and for individuals at high risk for CAD [9].

\section{Molecular Genetics}

FH is caused by loss of function mutations in the lowdensity lipoprotein receptor $(L D L R)$ gene, mutations in apolipoprotein B-100 (APOB) that affect the LDL receptorbinding domain, or gain of function mutations in the proprotein convertase subtilisin/kexin 9 (PCSK9) gene. LDLR genetic testing has been shown to identify $70-80 \%$ of individuals with a "definite" FH clinical diagnosis and 20-30\% with a less certain diagnosis [6]. Over 1,000 mutations in $L D L R$ have been identified in FH patients [10]. There is evidence that null mutations in $L D L R$ are associated with a more severe phenotype $[11,12]$. It has been estimated that mutations in $A P O B$ account for approximately $5.5 \%$ of $\mathrm{FH}$ cases, while mutations in PCSK 9 account for approximately $1.5 \%$ [10].

\section{Through a Public Health Lens}

It is estimated that there are over 620,000 patients with FH in the US, with less than $1 \%$ diagnosed [1 $\bullet$ ]. Four years ago, Dr. Paul Hopkins stated it best when he wrote, "FH is the only genetic cause of premature CAD for which a systematic, population-based approach to find affected individuals and screen their families is clearly warranted at this time" [13]. The Centers for Disease Control and Prevention Office of Public Health Genomics has responded and recently classified cascade testing for FH as a "tier 1" genomic application, meaning the action should be implemented in practice [14]. Universal cholesterol screening for children ages 9-11 years has been recommended by a National Lipid Association (NLA) Expert Panel on FH [15••] and a National Heart, Lung, and Blood Institute (NHLBI) Expert Panel on Cardiovascular Health and Risk Reduction in Children and Adolescents; the NHLBI guidelines have also been endorsed by the American Academy of Pediatrics [16]. For children with a family history of premature coronary heart disease (men $<55$ years, women $<65$ years) or elevated cholesterol, cholesterol screening should be considered beginning at age 2 years [15••]. For all individuals with cholesterol levels suspicious for $\mathrm{FH}$, the NLA recommends a family history of high cholesterol and heart disease in first-degree relatives be collected [15••].

\section{Role of the Genetic Counselor in the FH Population}

Genetic counseling is the process of helping individuals and their families understand and adapt to the medical, psychological, and familial implications of a genetic condition [17]. "This process integrates: (1) interpretation of family and medical histories to assess the chance of disease occurrence or reoccurrence, (2) education about inheritance, testing, management, prevention, resources, and research, and (3) counseling to promote informed choices and adaptation to the risk or condition" [17]. Genetic counseling has been recognized by physicians for its many positive contributions for patients and families with inherited forms of heart disease [18] and is indicated regardless of whether or not genetic testing will be performed. Multiple genetic counseling service delivery models exist, including in-person, telephone, group, and telegenetic, as well as varied types of referral patterns of patients for genetic counseling [19].

Genetic counselors are health care professionals with specialized graduate degrees and expertise in medical genetics and counseling. Many genetic counselors work as members of a health care team, and they provide information and support to families with inherited conditions. Cardiovascular genetic counselors are an important resource and integral health care team member for patients and families with inherited heart disease, including those families that have suffered a sudden death in a young person, which can occur in families with FH [20, 21]. It has been suggested that a master's-trained, board-certified genetic counselor, preferably with specialized training in cardiovascular genetics, be part of the multidisciplinary 
Table 1 The genetic counseling process for patients with $\mathrm{FH}$

Collection of $\geq 3$ generation family medical history information (pedigree), with special attention to "red flags" for $\mathrm{FH}$

"Red flags" for FH in the pedigree include hypercholesterolemia; premature CAD (onset in men before age 55 years and women before age 65 years) including angina pectoris and myocardial infarction; sudden cardiac death; physical features of FH (e.g., xanthomas, corneal arcus)

Because patients' self-reported family history information can have both reduced sensitivity and specificity, it is important to collect medical records, autopsy reports, and death certificates when possible so that diagnoses can be confirmed

In some cases, it may not be until clinical screening commences through the family that $\mathrm{FH}$ can be diagnosed

Family history is not static, but changes over time, and should therefore be updated periodically

Performance of risk assessment utilizing medical and family history information

Discussion of mode of inheritance and recurrence risk to family members

Facilitation of genetic testing

Pre- and post-test genetic counseling

Disclosure and documentation of genetic testing results

Facilitation of family-based care

Cascade testing

Discussion of screening, prevention, and medical management options in conjunction with managing physician

Discussion of reproductive options

Provision of written documentation of medical, genetic, and counseling information to referring health care providers and patients, including "Dear Family Member Letters"

Provision of psychosocial counseling and anticipatory guidance

Provision of education and resources from national organizations and advocacy groups

Discussion of available research study options

For example, enrolling FH patients into the CASCADE FH Registry

Discussion of the availability of DNA banking, when applicable

team involved in the care of families with heritable cardiovascular diseases [22]. An integrated approach that includes a genetic counselor may be associated with better adjustment and less worry in patients with inherited forms of heart disease [23]. Genetic counselors can be located by utilizing the "Find a Genetic Counselor" tool on the National Society of Genetic Counselors (NSGC) website, www.nsgc.org.

The genetic counseling process for patients with $\mathrm{FH}$ should include multiple components, listed and explained in further detail in Table 1. Insight and guidance on the implementation of a cardiovascular genetic medicine program and the potential psychological issues that may need to be addressed in the setting of genetic testing for inherited cardiovascular diseases have been provided previously [24, 25].

\section{Genetic Testing Should be Accompanied by Pre- and Post-Test Genetic Counseling}

Genetic testing is the gold standard for $\mathrm{FH}$ diagnosis since detection of a pathogenic mutation in $L D L R, A P O B$, or $P C S K 9$ provides an unequivocal diagnosis [26]. In the US, the NLA Expert Panel on FH published recommendations that includes a statement that FH genetic testing should be covered by payers when the diagnosis of $\mathrm{FH}$ is uncertain [27]. The International FH Foundation recently published a consensus-based guidance document on the care of $\mathrm{FH}$, which was generated from a series of seminars and workshops held at an international symposium attended by $\mathrm{FH}$ experts. This document states that genetic testing for $\mathrm{FH}$ should ideally be offered to all index cases who have a phenotypic diagnosis of $\mathrm{FH}$; this is a level $3 \mathrm{~A}$ recommendation (level of evidence is "expert opinion or clinical experience") [28••]. This same guideline recommends (level 1A) that pre-test counseling should be offered to atrisk family members prior to any form of testing [28••]. Pre-test counseling is also recommended in the 2013 Consensus Statement of the European Atherosclerosis Society on FH [1].

Pre-test genetic counseling should include information on the indications for genetic testing as well as a discussion regarding the benefits, limitations, familial implications, and potential risks of genetic testing to insure informed decision-making. Pre-test counseling is also indicated in order to inform $\mathrm{FH}$ patients undergoing genetic testing of the multiple possible types of results they may receive. While a pathogenic mutation may be identified, it is also possible that previously reported and/or novel variants of uncertain significance and/or benign variants may be found. Online, open-access databases listing DNA variants with annotated references are valuable to the interpretation of variants, and such a database exists for $L D L R$ [29]. Both laboratory-based and clinical genetic counselors are valuable resources in the interpretation and explanation of complex genetic testing results [30].

$\mathrm{FH}$ genetic testing may also be negative, and it is imperative for patients with a clinical phenotype of $\mathrm{FH}$ to understand that undetected mutations as well as mutations in untested and/or as-yet-undiscovered genes cannot be excluded. A 2013 study conducted by Hilgart et al. [31] examined individuals' responses to negative $\mathrm{FH}$ genetic testing results and found that feelings of uncertainty were exacerbated by lack of information and that these patients felt they would benefit from discussing their result with a health professional. The findings of this study suggest that it would be helpful for patients to be counseled by a clinician prior to testing about the possibility of a negative $\mathrm{FH}$ genetic testing result and also to be provided with information regarding what a negative result means regarding 
their condition's cause and how their result impacts their children.

A complete analysis of all known FH genes, including full gene sequencing and large rearrangement testing, provides the highest clinical sensitivity of genetic testing. This is particularly important in families where LDL-C values vary greatly, as there may be more than one gene mutation in the family [32]. Clinical genetic testing is available in the US via commercial genetic testing laboratories with ranging technologies, clinical sensitivities, costs, and the ability to bill health insurance. Up-to-date information on clinical and research genetic testing options can be located in two online genetic testing databases: the National Institutes of Health Genetic Testing Registry (http://www.ncbi.nlm.nih.gov/gtr) and GeneTests (genetests.org).

The clinical sensitivity of current $\mathrm{FH}$ genetic testing is not $100 \%$. Targeted genetic screening (e.g., focusing on pediatric populations) has been shown to lead to higher (95\%) mutation detection rates [33]. Clinical sensitivity is likely even lower in patients of varied ethnic backgrounds [34]. Technological advances, including next generation and whole exome sequencing, will likely increase the costeffectiveness and clinical sensitivity of $\mathrm{FH}$ genetic testing $[35,36]$. It has also been suggested that a substantial number of patients with a clinical diagnosis of $\mathrm{FH}$, but without a pathogenic variant in one of the three main known genes, may have elevated LDL-C levels due to a polygenic etiology [37].

Genetic testing may also begin to play a larger role with therapeutic decision-making in both $\mathrm{HeFH}$ and $\mathrm{HoFH}$ patients. For example, the effectiveness of certain therapies, such as the proprotein convertase subtilisin/kexin 9 monoclonal antibody, AMG 145, depended upon the HoFH patient's genotype in a recent study, with no LDL-C response seen in the two patients in this trial who were LDL receptor negative [38].

Genetic testing results can also impact family dynamics and relationships. Parents in particular may have feelings of guilt related to passing their mutation to their children. In this situation, it may be helpful to emphasize the benefits provided by this type of genetic information (i.e., knowledge is power); specifically, clinicians can utilize this information to initiate clinical screening that may lead toward the earliest possible detection of disease and that may provide the opportunity for earlier treatment and lifestyle modifications [39].

\section{Cascade Screening Implementation}

Cascade screening is a mechanism for identifying people at risk for a genetic condition by a process of systematic family tracing. It should begin with first-degree relatives (parents, siblings, children) and then extend to second- and third-degree relatives in a stepwise cascade fashion, moving through the pedigree in sequential steps as additional family members are diagnosed and until all at-risk relatives have been identified. Cascade screening for $\mathrm{FH}$ has been shown to be a cost-effective method for identifying new cases of FH [40-42]. DNA testing should be utilized for $\mathrm{FH}$ cascade screening if the pathogenic mutation has been identified in the index case [28••]. Once the disease-causing mutation has been identified, cascade genetic testing allows for the definitive diagnosis of $\mathrm{FH}$ in at-risk relatives (100\% positive predictive value).

Cascade screening is not effective unless at-risk biological relatives of the $\mathrm{FH}$ index patient can be identified and notified of their risk, health implications, and the availability of FH testing. However, the best means by which to contact relatives of the $\mathrm{FH}$ index patient in order to proceed with systematic cascade screening is not entirely clear because of ethical factors and family dynamics, including the maintenance of confidentiality and privacy, 'duty to warn,' potential for psychological harm, and right 'not to know,' among others [43]. It is recommended that a clinician construct a family pedigree, identify relatives who should be offered FH testing, and discuss the notification of at-risk relatives with the index case [28••]. This is a standard part of the genetic counseling process. It is also recommended that index cases be provided with written information and encouraged to share this with relatives. Such "Dear Family Member Letters" have been shown to be an effective way to inform relatives about inherited cardiac disease [44] and should include general information about $\mathrm{FH}$ and emphasize health consequences without testing and the benefit and availability of preventive therapies [28••].

\section{Raising Awareness}

In 2011, a survey of cardiologists was conducted by the American College of Cardiology (ACC) that indicates a need for increased awareness of $\mathrm{FH}$ among cardiologists regarding how common it is, its heritability, and the risk of cardiovascular disease associated with FH [45]. Many organizations are putting a heavy focus on raising awareness of FH including the NLA (lipid.org), whose scientific sessions conference in 2014 is focused specifically on $\mathrm{FH}$ and is titled "New Era in Lipidology: Familial Hypercholesterolemia, Guidelines and Risk Assessment in the Clinic" and includes a session titled "Role of Genetic Counseling in FH: What Referring Physicians and Patients Need to Know." Also, the Foundation of the NLA has developed extensive patient educational information 
including a website called "Learn Your Lipids" (learnyourlipids.com). The NLA has also developed a PocketGuide on FH for health care providers that includes information on diagnosis, screening, and treatment and can be located on GuidelineCentral.com. The FH Foundation (thefhfoundation.org) is a patient-centered nonprofit organization dedicated to education, advocacy, and research of FH. In order to address gaps in knowledge and barriers to FH screening, identification, and treatment, The FH Foundation recently established a national registry for $\mathrm{FH}$ patients in the US: CAscade SCreening for Awareness and DEtection of FH (CASCADE FH) [46•]. As the only active US-based registry for FH, CASCADE FH aims to promote cascade screening implementation, more timely disease identification, and optimal treatment to improve clinical outcomes and quality of life for all $\mathrm{FH}$ patients [46*]. The FH Foundation also held an International FH Summit in September 2013 in Annapolis, Maryland, and 24 September 2013 was the 2nd Annual National FH Awareness Day. In addition, the Preventive Cardiovascular Nurses Association (PCNA) has also been actively working to expand awareness of $\mathrm{FH}$ [47] and has several $\mathrm{FH}$ educational resources on their website (pcna.net). The National Society of Genetic Counselors has also developed an official Working Group on FH within their Cardiovascular Genetics Special Interest Group, who have developed resources such as a "Dear Family Member Letter," available on their website (nsgc.org) and also as a downloadable file under the Resources section of The FH Foundation's website.

\section{Conclusions}

Master's-trained genetic counselors, specialized health care professionals with expertise in genetics and counseling, have been recommended as a solution for the incorporation of genetic medicine by providing genetic counseling and testing to applicable patients and by working in concert with subspecialty physicians [48]. A timely American Heart Association policy statement on genetics and cardiovascular disease strongly advocates for the involvement of centers with expertise in cardiovascular genetics to guide the appropriate initiation, interpretation, and implementation of genetic testing [49]. A pedigree should be collected, assessed, and stored on every patient with FH. Cascade testing needs to be performed and index patients should be provided with tools to assist them in informing their at-risk relatives about FH. Families with FH may face different types of psychological issues, and genetic counselors are available as a resource to help them work through and hopefully overcome potential difficult dilemmas and family dynamics. In conclusion, genetic counselors can help win the "FH battle." Because, as Newson and Humphries [43] so eloquently stated, "Our biology does not stop: the risk of developing coronary heart disease as a consequence of FH will still be present, even if relatives live in ignorance."

Disclosure AC Sturm declares no conflicts of interest.

Human and Animal Rights and Informed Consent This article does not contain any studies with human or animal subjects performed by any of the authors.

\section{References}

Papers of particular interest, published recently, have been highlighted as:

- Of importance,

•- Of major importance

1. • Nordestgaard BG, Chapman MJ, Humphries SE et al. Familial hypercholesterolemia is underdiagnosed and undertreated in the general population: guidance for clinicians to prevent coronary heart disease: consensus statement of the European Atherosclerosis Society. Eur Heart J. 2013;34(45):3478-3490a. Recent consensus statement that provides in-depth review of $\mathrm{FH}$ prevalence, screening strategies, diagnosis and treatment with multiple informative figures and tables.

2. Alonso R, Mata P, Zambon D, Mata N, Fuentes-Jimenez F. Early diagnosis and treatment of familial hypercholesterolemia: improving patient outcomes. Exp Rev Cardiovasc Ther. 2013; 11(3):327-42.

3. Slack J. Risks of ischaemic heart-disease in familial hyperlipoproteinaemic states. Lancet. 1969;2(7635):1380-2.

4. Williams RR, Hunt SC, Schumacher MC, et al. Diagnosing heterozygous familial hypercholesterolemia using new practical criteria validated by molecular genetics. Am J Cardiol. 1993;72(2):171-6.

5. Organization WHO. Familial hypercholesterolemia. Report of a second WHO consultation. 1999.

6. DeMott K, Nherera L, Shaw EJ, Minhas R, Humphries SE, Kathoria M, Ritchie G, Nunes V, Davies D, Lee P, McDowell I, Neil A, Qureshi N, Rowlands P, Seed M, Stracey H, Thorogood $\mathrm{M}$, Watson M. Clinical guidelines and evidence review for familial hypercholesterolemia: the identification and management of adults and children with familial hypercholesterolemia. London: Royal College of General Practitioners; 2008.

7. Robinson JG. Management of familial hypercholesterolemia: a review of the recommendations from the National Lipid Association Expert Panel on familial hypercholesterolemia. J Manag Care Pharm. 2013;19(2):139-49.

8. Rader DJ, Kastelein JJ. Lomitapide and mipomersen: two first-inclass drugs for reducing low-density lipoprotein cholesterol in patients with homozygous Familial hypercholesterolemia. Circulation. 2014;129(9):1022-32.

9. Toth PP. Emerging LDL therapies: mipomersen-antisense oligonucleotide therapy in the management of hypercholesterolemia. J Clin lipidol. 2013;7(3 Suppl):S6-10.

10. Varret M, Abifadel M, Rabes JP, Boileau C. Genetic heterogeneity of autosomal dominant hypercholesterolemia. Clin Genet. 2008;73(1):1-13. 
11. Vilades Medel D, Leta Petracca R, Carreras Costa F, et al. Coronary computed tomographic angiographic findings in asymptomatic patients with heterozygous familial hypercholesterolemia and null allele low-density lipoprotein receptor mutations. Am J Cardiol. 2013;111(7):955-61.

12. Alonso R, Mata N, Castillo S, et al. Cardiovascular disease in familial hypercholesterolemia: influence of low-density lipoprotein receptor mutation type and classic risk factors. Atherosclerosis. 2008;200(2):315-21.

13. Hopkins PN. Defining the challenges of familial hypercholesterolemia screening: introduction. J Clin Lipidol. 2010;4(5): $342-5$.

14. Khoury MJ, Coates RJ, Evans JP. Evidence-based classification of recommendations on use of genomic tests in clinical practice: dealing with insufficient evidence. Genet Med. 2010;12(11): $680-3$.

15. • Goldberg AC, Hopkins PN, Toth PP et al. Familial hypercholesterolemia: screening, diagnosis and management of pediatric and adult patients: clinical guidance from the National Lipid Association Expert Panel on Familial Hypercholesterolemia. J Clin Lipidol. 2011;5(3 Suppl):S1-8. This executive summary from the NLA provides comprehensive clinical guidance including information on universal and cascade screening, diagnosis, and treatment recommendations for adults and pediatric patients.

16. Expert Panel on Integrated Guidelines for Cardiovascular Health and Risk Reduction in Children and Adolescents; National Heart, Lung, and Blood Institute. Expert panel on integrated guidelines for cardiovascular health and risk reduction in children and adolescents: summary report. Pediatrics. 2011;128 (Suppl 5):S213-256

17. Resta R, Biesecker BB, Bennett RL, et al. A new definition of genetic counseling: National Society of Genetic Counselors' Task Force report. J Genet Couns. 2006;15(2):77-83.

18. Patterson C. A good idea: a physician's perspective on genetic counseling for hypertrophic cardiomyopathy. J Cardiovasc Transl Res. 2009;2(4):508-9.

19. Cohen SA, Marvin ML, Riley BD, Vig HS, Rousseau JA, Gustafson SL. Identification of genetic counseling service delivery models in practice: a report from the NSGC Service Delivery Model Task Force. J Genet Couns. 2013;22(4):411-21.

20. Ingles J, Yeates L, Semsarian C. The emerging role of the cardiac genetic counselor. Heart Rhythm. 2011;8(12):1958-62.

21. DE Macleod H, Honeywell C, Rutberg J. Genetic counselors: an important resource for families following a young sudden cardiac death. Acad Forensic Pathol. 2013;3(2):183-90.

22. Tester DJ, Ackerman MJ. Genetic testing for potentially lethal, highly treatable inherited cardiomyopathies/channelopathies in clinical practice. Circulation. 2011;123(9):1021-37.

23. Ingles J, Lind JM, Phongsavan P, Semsarian C. Psychosocial impact of specialized cardiac genetic clinics for hypertrophic cardiomyopathy. Genet Med. 2008;10(2):117-20.

24. Sturm AC, Hershberger RE. Genetic testing in cardiovascular medicine: current landscape and future horizons. Curr Opin Cardiol. 2013;28(3):317-25.

25. Aatre RD, Day SM. Psychological issues in genetic testing for inherited cardiovascular diseases. Circ Cardiovasc Genet. 2011; 4(1):81-90.

26. Humphries SE, Norbury G, Leigh S, Hadfield SG, Nair D. What is the clinical utility of DNA testing in patients with familial hypercholesterolemia? Curr Opin Lipidol. 2008;19(4):362-8.

27. Goldberg AC, Robinson JG, Cromwell WC, Ross JL, Ziajka PE. Future issues, public policy, and public awareness of familial hypercholesterolemias: recommendations from the National Lipid Association Expert Panel on familial hypercholesterolemia. J Clin Lipidol. 2011;5(3 Suppl):S46-51.
28. • Watts GF, Gidding S, Wierzbicki AS et al. Integrated guidance on the care of familial hypercholesterolemia from the International FH Foundation. Int J Cardiol. 2014:171(3):309-325. Provides consensus-based guidance from a team of international $F H$ experts including recommendations on the detection, diagnosis, assessment, management of FH in adults and children, as well as best practices for cascade screening, risk notification, and testing of families with $\mathrm{FH}$, including the use of genet testing.

29. Usifo E, Leigh SE, Whittall RA, et al. Low-density lipoprotein receptor gene familial hypercholesterolemia variant database: update and pathological assessment. Ann Hum Genet. 2012; 76(5):387-401.

30. Caleshu C, Day S, Rehm HL, Baxter S. Use and interpretation of genetic tests in cardiovascular genetics. Heart. 2010;96(20): $1669-75$.

31. Hilgart J, Mercer J, Thirlaway K. Individuals' experiences of, and responses to, a negative genetic test result for familial hypercholesterolemia. J Health Psychol. 2013;18(3):339-49.

32. Soufi M, Rust S, Walter M, Schaefer JR. A combined LDL receptor/LDL receptor adaptor protein 1 mutation as the cause for severe familial hypercholesterolemia. Gene. 2013;521(1):200-3.

33. Van Der Graaf A, Avis HJ, Kusters DM, et al. Molecular basis of autosomal dominant hypercholesterolemia: assessment in a large cohort of hypercholesterolemic children. Circulation. 2011; 123(11):1167-73.

34. Ahmad Z, Adams-Huet B, Chen C, Garg A. Low prevalence of mutations in known loci for autosomal dominant hypercholesterolemia in a multiethnic patient cohort. Circ Cardiovasc Genet. 2012;5(6):666-75.

35. Motazacker MM, Pirruccello J, Huijgen R, et al. Advances in genetics show the need for extending screening strategies for autosomal dominant hypercholesterolemia. Eur Heart J. 2012;33(11): 1360-6.

36. Vandrovcova J, Thomas ER, Atanur SS, et al. The use of nextgeneration sequencing in clinical diagnosis of familial hypercholesterolemia. Genet Med. 2013;15(12):948-57.

37. Talmud PJ, Shah S, Whittall R, et al. Use of low-density lipoprotein cholesterol gene score to distinguish patients with polygenic and monogenic familial hypercholesterolemia: a casecontrol study. Lancet. 2013;381(9874):1293-301.

38. Stein EA, Honarpour N, Wasserman SM, Xu F, Scott R, Raal FJ. Effect of the proprotein convertase subtilisin/kexin 9 monoclonal antibody, AMG 145, in homozygous familial hypercholesterolemia. Circulation. 2013;128(19):2113-20.

39. Ingles J, Zodgekar PR, Yeates L, Macciocca I, Semsarian C, Fatkin D. Guidelines for genetic testing of inherited cardiac disorders. Heart, Lung Circ. 2011;20(11):681-7.

40. Marks D, Wonderling D, Thorogood M, Lambert H, Humphries SE, Neil HA. Screening for hypercholesterolemia versus case finding for familial hypercholesterolemia: a systematic review and cost-effectiveness analysis. Health Technol Assess. 2000;4(29):1-123.

41. Marks D, Wonderling D, Thorogood M, Lambert H, Humphries SE, Neil HA. Cost effectiveness analysis of different approaches of screening for familial hypercholesterolemia. BMJ. 2002;324(7349): 1303 .

42. Nherera L, Marks D, Minhas R, Thorogood M, Humphries SE. Probabilistic cost-effectiveness analysis of cascade screening for familial hypercholesterolemia using alternative diagnostic and identification strategies. Heart. 2011;97(14):1175-81.

43. Newson AJ, Humphries SE. Cascade testing in familial hypercholesterolemia: how should family members be contacted? Eur J Hum Genet. 2005;13(4):401-8.

44. Van Der Roest WP, Pennings JM, Bakker M, Van Den Berg MP, Van Tintelen JP. Family letters are an effective way to inform relatives about inherited cardiac disease. Am J Med Genet A. 2009;149A(3):357-63. 
45. Foody JM. Familial Hypercholesterolemia: an under-recognized but significant concern in cardiology practice. Clin Cardiol. 2014;37(2):119-25.

46. - O'Brien EC, Roe MT, Fraulo ES et al.: Rationale and design of the familial hypercholesterolemia foundation CAscade SCreening for Awareness and DEtection of Familial Hypercholesterolemia registry. Am Heart J. 2014;167(3):342-349, e317. Provides information on the CASCADE FH Registry, including its objectives, design, inclusion criteria, methods of enrollment, and data elements collected.
47. Ross J. Educating patients about familial hypercholesterolemia: the role of the cardiovascular nurse. $J$ Cardiovasc Nurs. 2013;28(2):102.

48. Hershberger RE. Cardiovascular genetic medicine: evolving concepts, rationale, and implementation. J Cardiovasc Transl Res. 2008;1(2):137-43.

49. Ashley EA, Hershberger RE, Caleshu C, et al. Genetics and cardiovascular disease: a policy statement from the American Heart Association. Circulation. 2012;126(1):142-57. 